\title{
Rede pan-americana de informação e documentação em engenharia sanitária e ciências do ambiente (REPIDISCA)
}

A REPIDISCA é um sistema regional de informação com a finalidade de disseminar informação disponível sobre Engenharia Sanitária e Ciências do Ambiente. A Rede inclui material bibliográfico, tendo interesse especial por relatórios gerados na América Latina e Caribe.

A REPIDISCA tem com Centro de Coordenação Regio. nal o Centro Pan-Americano de Engenharia Sanitária e Ciências do Ambiente (DEPIS), sediado em Lima - Peru, e faz parte do Programa de Saúde Ambiental da Organização Pan-Americana da Saúde (OPAS). É patrocinada pelo Centro Internacional de Investigação para o Desenvolvimento (CIID) do Canadá, pela UNESCO e pelo Centro Internacional de Referência para Abastecimento Público de Água e Saneamento da Holanda.

Duzentos Centros Cooperantes da Rede trabalham indentificando informação concernente à Engenharia Sanitária e Ciências do Ambiente, disponível nas bibliotecas, centros de documentação e outros. No Brasil, a Secretaria Especial do Meio Ambiente (SEMA) atua como Centro de Coordenação Nacional, contando, atualmente, com a elaboração ${ }^{\circ}$ de 40 Centros Cooperantes e tendo a Companhia de Tecnologia e Saneamento Ambiental (CETESB) como Centro de Coordenação para São Paulo e a Região Sul.

Serviços e produtos da Rede:

a) Base de dados Bibliográficos:

- REPINDEX (publicação trimestral, que oferece, em cada número, 700 resumos de documentos, que podem ser obtidos através de fotocópias ou microficha).

- Catálogo Coletivo de Publicações Periódicas.

- Catálogo da Biblioteca do CEPIS.

- Buscas bibliográficas correntes e retrospectivas.

- Fitas magnéticas e disquetes.

b) Base de Dados não-Bibliográficos:

- Diretórios e Guias.

c) Outros Serviços:

- Sumários de revistas (TABCONT/CEPIS).

- Informações sobre cursos, reuniões, seminários, etc.

CENTROS COOPERANTES DA REPIDISCA NO BRASIL

ADEMA (SE)

$\mathrm{BNH}(\mathrm{RJ})$ 
CASAN (SC)

CESP (SP)

CETEC (MG)

CETESB (SP)

CETREL (BA)

COMLURB (RJ)

COMPESA (PE)

CONDER (BA)

COPASA (MG)

COPEC (BA)

CORSAN (RS)

CRA (BA)

DMA (RS)

DMAE (RS)

DNAEE/DCRH (DF)

DNOS (RJ)

DNPM (DF)

EMBASA (BA)

ENGE-RIO (RJ)

FAC. SAUUDE PÚBLICA (SP)

ESCOLA NACIONAL DE SAÚDE PÚBLICA (RJ)

FATMA (SC)

FEEMA (RJ)

FUNDACENTRO (SP)

FUNDREM (RJ)

IBDF (DF)

IDEC (RN)

INT/FTI (RJ)

ISAM (PR)

MIN. SAUDE (DF)

SABESP (SP)

SANEPAR (PR)

SEMA (DF)

SEPLANTEC/CEI (BA)

SMAM (RS)

SUREHMA (PR)

UFBA (BA)

UNB (DF)

USP/EESC (SP)

IBAM (RJ)

FSESP (RJ)

Informações: Organização Pan-Americana da Saúde (OPAS) Centro Pan-Americano de Engenharia Sanitária e Ciências do Ambiente, Lima - Peru. 